\title{
Design of Simulation Model on the Battlefield Environment ZHANG Jianli ${ }^{1,}$,, ZHANG Lin $^{2}{ }^{\text {b }}$, JI Lijian $^{1, ~ c}$, GUO Zhongwei ${ }^{1, d}$ \\ ${ }^{1}$ Army Officer Academy of PLA,Hefei,230031,Anhui, China \\ ${ }^{2}$ Dept. of Computer Engineering ,Anhui SanLian University,Hefei,230000,Anhui,China \\ awjxc1982@126.com bsirenrabbit@sina.com dguo_zw@126.com
}

Keywords: Digital earth, Variable resolution technology, Particle system

\begin{abstract}
In order to implement the informatization under the condition of forces global training requirements, the paper constructed the basic process of the battlefield environment simulation model, including geographic simulation model, simulation models of meteorological and electromagnetic environment simulation model. Virtual training for the combat troops have provided the certain basis.
\end{abstract}

\section{Introduction}

In order to meet the information needs of global training for forces, it must build the battlefield environment simulation model. The digital elevation model (DEM) and mass, multi-source geographic data and remote sensing digital image overlay, generated with the actual topography, geomorphology, the matching of the battlefield environment, such as coordinates, elevation, support rapid space measurement, meteorology, electromagnetic environment visualization, adapt to the modern condition, the forces on the mountain and plain, need to wait for all sorts of complex terrain in a coastal area. The paper mainly studied the basic process to construct geographic simulation model, simulation models of meteorological and electromagnetic environment simulation model.

\section{The Geographical Simulation Model}

Geographic simulation environment should be able to support a virtual browse, three-dimensional point, query place name , accurate positioning, distance measurement, area measurement, visibility analysis, and other functions, and can be deployed combat forces and support electronic sand table deduction, etc.

Building Digital Earth. Digital earth is a based on geographic coordinates (longitude and latitude), multi-resolution, huge amounts of data and multi-dimensional display of virtual system, it requires that all the information on the digital earth.

(1) Build the coordinate system

Based on mathematical model of WGS84 coordinate system is a sphere, as shown in figure 1. The world of any point on a sphere coordinates can be calculated by the following formula:

double $X=R \times \cos ($ lat $) \times \cos ($ lon $)$

double $Y=R \times \cos ($ lat $) \times \sin ($ lon $)$

double $Z=R \times \sin ($ lat $)$

$R$ for any radius, lat for this point of latitude (in radians), lon for the longitude of the point where (in radians). 


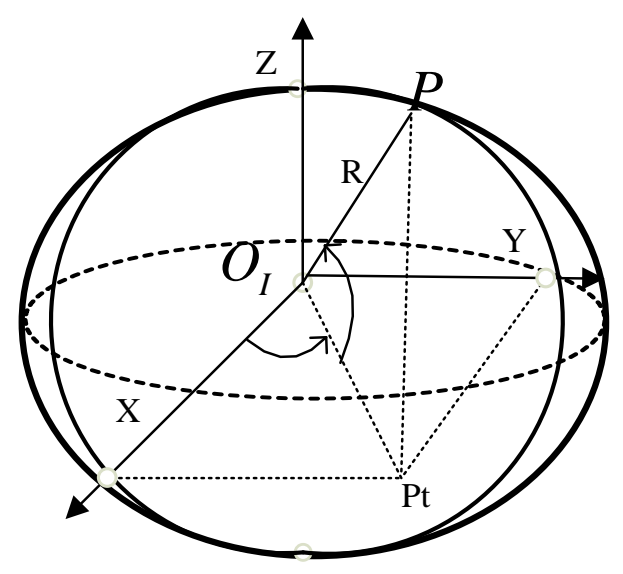

Figure 1 WGS84 coordinate system schematic diagram

(2) Build a space object height model

The height of the spatial object model is divided into five kinds: relative to the ground, the sea bottom in the relatively, attached to the ground, the sea bottom in the attachment, and absolute height, through these five high spatial model can accurately determine the coordinates of the object, so that you can express, air, sea, sea surface and the surface under various objects.

(3)Build a level of detail (LOD) model

Level of detail model is established on the basis of massive amounts of data and storage. Firstly, in accordance with the geographic coordinates of regional classification it don't number LOD data structure; Secondly, in accordance with the LOD to physical re-sampling of basic data, data structure to establish various other data files, the higher level the more data; Finally according to the regional the distance from viewpoint to load, the higher the viewpoint, the lower load data, data point of view, the lower load level is higher.

From Figure 2 we can see by the superposition of satellite remote sensing images and digital elevation model surface features, which is formed by the way of the real land form environment.

(4) Texture mapping

Texture mapping as a kind of commonly used method of adding surface detail for the virtual $3 \mathrm{D}$ objects, is one of important research field in computer graphics, to show the object's color, texture play a key role. According to the different generating method ,it can be divided into surface texture to texture and texture two entities. Surface texture is generated through texture mapping. Mathematically speaking, it is a $2 \mathrm{D}$ image to the mapping of the space surface, namely the plane with characteristics of material texture image "package" onto the surface of the three-dimensional objects.

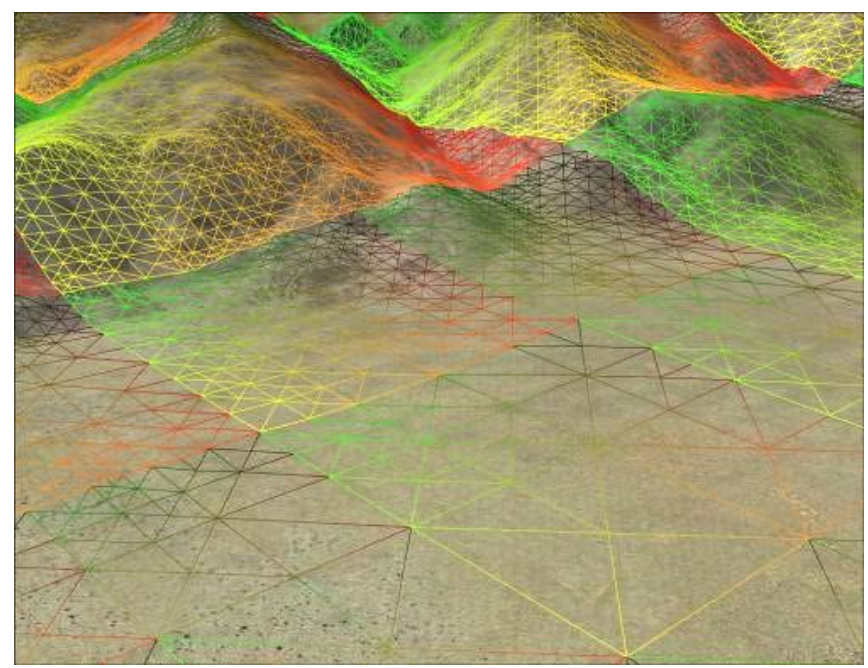

Figure 2 rendering of large-scale terrain 
Geographic Data And Model Design. On the basis of digital earth, scenario uses integrated large-scale, vast, multi-source data based image data, and the world to quickly build three-dimensional geographic space.

(1) Design Relational database

Using object-oriented data model and abstract methods, using universal modeling language (UML), testing the semantic correctness of logical architecture and integrity, with clear rules, can be instantiated object data model. Using this database retrieval model quickly, improve the efficiency of handling of geographic data, allow them to quickly retrieve and update the display real-time geographic information.

(2) Design Multilayer visual variable resolution control

To meet the needs of different hierarchical levels on the natural environment model, using a resolution modeling technology for different application layer according to proportion, conduct the thorough research to the show details, and so on, so as to make the system can dynamically according to the need of situation change resolution, effectively solve the massive terrain data and display the contradiction of limitation. In specific design, the integration of Hierarchical Variable Resolution technology (Integrated Hierarchical Variable Resolution, IHVR) achieve the design requirements. IHVR is a hierarchical decomposition method for parameters of the process. Model input parameters can be according to the level of change, high-level parameters can be input directly, can also be obtained by low-level operation parameters. Can be seen from figure 3 , in each resolution level, the entity, process, decision-making and control all have this similar exercise the function of the recursive call. In programming, therefore, the process is divided into two kinds, function process and control process of two kinds of process with common public parent class, the realization of the control process is based on the function of the lower level of process.

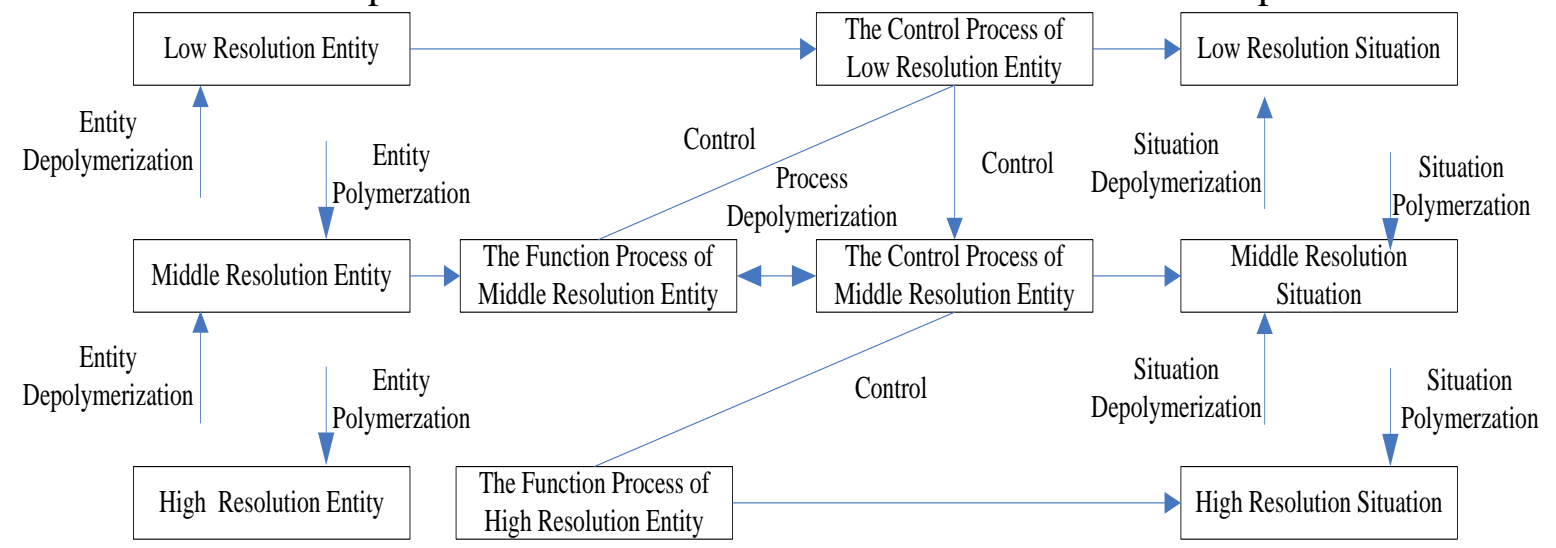

Figure 3 variable resolution process control level based on IHVR

(3) Design 3D terrain reconstruction and renewal

In order to improve the speed of data loading, prepossessing, image, DEM, vector data after pretreatment of data as a cache on the server, so that you can speed up the data download. At the same time the client also uses caching technology, namely through an area, the procedure to detect local cache first whether there is a regional data, if there is the direct use of the data in the local cache, otherwise the request to the server for the area of data, the server sends the data to the client after receiving the request for the use of application, the client receives the data at the same time, the data in a cache, so next time browsing this area can directly use the data in the cache, thus saved network round trip time, thus greatly improve the efficiency.

\section{Weather simulation model}

Vivid natural environment in accordance with regional characteristics (rain, thunder and lightning, snow, fog) can make the simulation scene more real, you can change the visibility of the battlefield simulation, to organize the implementation of the battlefield reconnaissance; observation burst point and target a certain degree of impact. Implement specific meteorological environment simulation, concrete is through the principle of particle system implementation. Particle system in a 
space area definition, and then change with time and parameters. These parameters include the motion path of a particle, color and shape. At a random time, it may remove every object. Particle shape may be small ball, ellipsoid, cube or other shape, particle size and shape change over time. Other properties such as particle transparency, color and movement will be random variation. Implementation of smoke based on particle system flow chart shown in figure 4.

Using particle system simulation of smoke process is as follows:

(1)The smoke particles are produced in the initial frame and the follow-up frame, and in subsequent frames in the sequence of each frame as long as the change in the initial particle properties.

(2) Due to the shape of the actual smoke can be varied, obviously it is impossible to completely simulate the actual situation. But it can be predefined by modeling a series of different shape of smoke, such as: triangles and rectangles, polyhedron, etc. There are 10 kinds of different shapes, such as now Numbers for them: $1,2, \ldots, 10$, and then use random function for explosive particles increase shape properties.

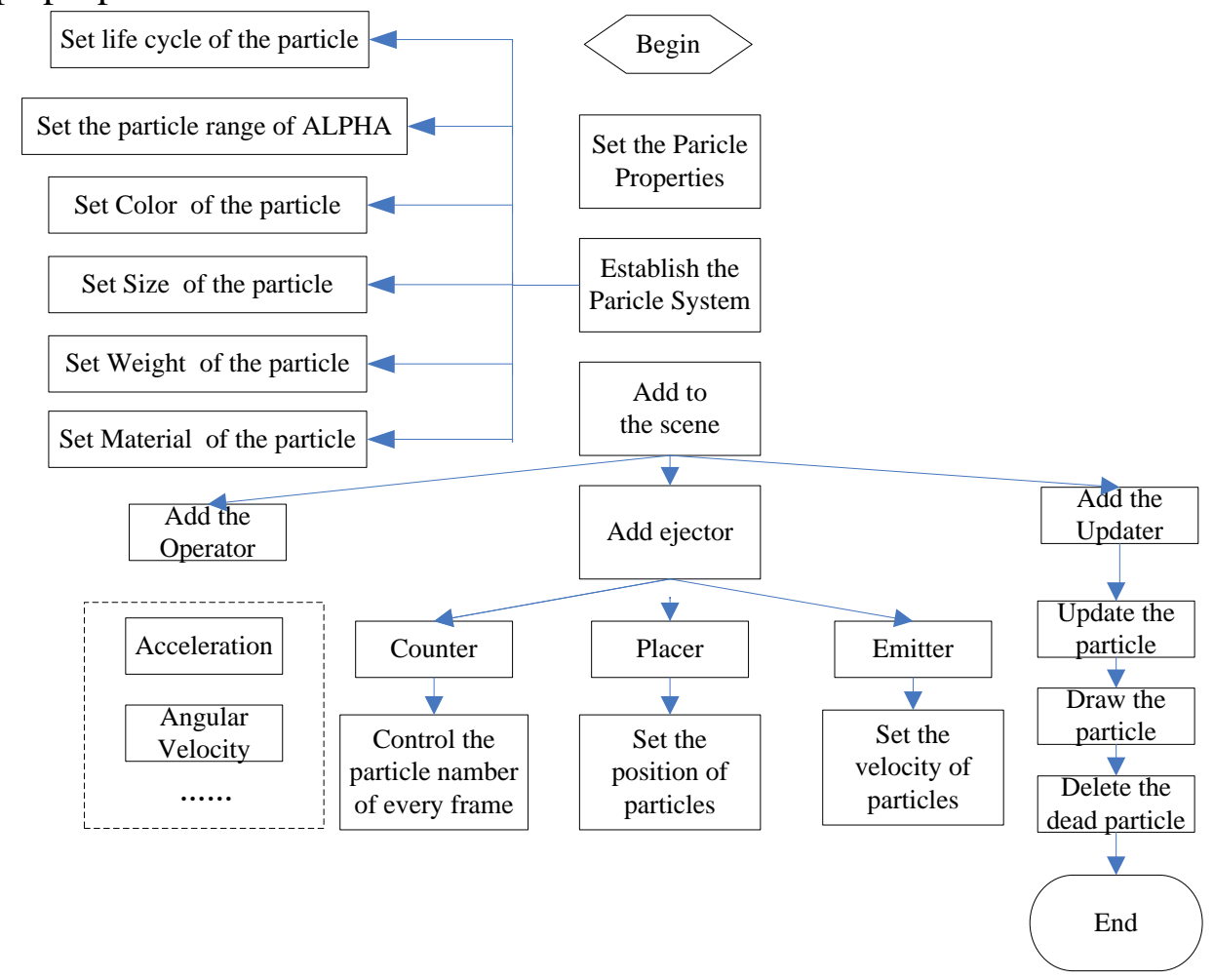

Figure 4 particle system flow chart of special effects

(3) In addition to the smoke movement in the process, the particles velocity in the direction of movement, and around the $x, y, z$ three axis of rotation. Assumes that particles and $x, y, z$ three axis forward into the Angle respectively: $p, h, r$ and around the three axis of rotation velocity is respectively: $w_{x} 、 w_{y}$ and $w_{z}, f_{i}$ is the first frame particles and $x, y, z$ three axis Angle for:

$$
\begin{aligned}
& p\left(f_{i}\right)=p(i-1)+w_{x} \times\left(f_{i}-f_{i-1}\right) \\
& h\left(f_{i}\right)=h(i-1)+w_{y} \times\left(f_{i}-f_{i-1}\right) \\
& r\left(f_{i}\right)=r(i-1)+w_{z} \times\left(f_{i}-f_{i-1}\right)
\end{aligned}
$$

(4) Due to the effect of gravity, the particle trajectory should conform to the actual situation of sport, until each frame of the particles.

\section{The electromagnetic environment simulation model}

Electromagnetic environment is the operational area of the sum total of all kinds of electromagnetic radiation source signal. In the modern war, especially in information warfare, the 
electromagnetic environment of battlefield plays a very important role. Describe the battlefield electromagnetic environment, can be more realistically, enables us to effectively study the law of modern warfare, conducts research methods, auxiliary battle command training, tactical commanders and staffs, and so on.

For example, In radar visualization system, it is mainly composed of virtual battlefield environment, detection range of radar three-dimensional data generation and detection range of radar three-dimensional visualization draw three parts, the specific system structure as shown in figure 5 .

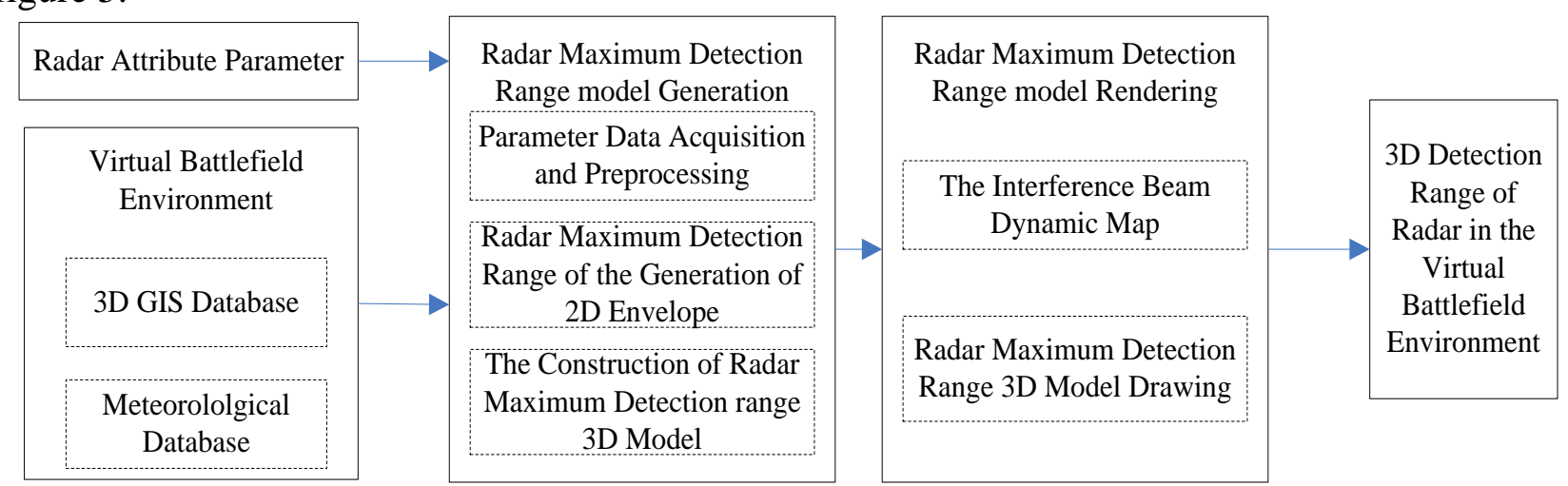

Figure 5 visualization system function diagram

Virtual battlefield environment contains the radar detection range model, terrain parameters of the radar detection range model .Radar detection range model generated mainly from obtain environmental terrain parameters in virtual battlefield environment, use of the user to enter the radar parameters, according to the radar equation under without interference and interference radar maximum detection range model was constructed.Radar maximum detection range model rendering performance is mainly the detection model in the virtual battlefield environment, and the integration of the various effects in radar range model drawing.

\section{Conclusion}

Research based on the global vector geographic information and imaging simulation of geographical environment, weather environment and the electromagnetic environment, under the condition of information can help to deepen the understanding of the complex battlefield environment, can carry on the bricks will force training, rich forces training means, virtual training for the combat troops have provided the certain basis.

\section{References}

[1] Johnson C.R., Top Scientific Visualization Research Problems, IEEE Computer Graphics and Applications, 2004, 24(4)

[2] C.N. JAMES, S.R. BRODZIK et al, Radar Data Processing and Visualization over Complex Terrain. Weather and Forecasting, 2000. 15(3)

[3] Zhong-feng wang, Yang Fengbao. Geodetic coordinate of the space rectangular coordinate transformation method [J]. Journal of surveying and mapping engineering, 2010, 12 (2)

[4] Liao Ming, Li peng, Chen Yiping. Geocentric rectangular coordinate and geodetic coordinate transformation algorithm study [J]. Journal of electronic science and technology, 2010, 23 (2)

[5]Hgues Hoppe.Smooth View-Dependent Level -of-Detail Control and its Application to Terrain Rendering.2008

[6]Karl D.Liebert. Using GIS vector data to build virtual environments for agent based models. Proceedings of 2008 Spring simulation conference. 2008 